\title{
Integrated automatic modular measuring system
}

\author{
I. D. Baikie, K. O. van der Werf, and L.J. Hanekamp \\ Department of Applied Physics, University of Twente, P.O. Box 217, 7500 AE Enschede, The Netherlands
}

(Received 19 October 1987; accepted for publication 20 May 1988)

\begin{abstract}
This paper describes a versatile automatic measuring system composed of discrete modules. The modules can operate in both stand-alone and remote modes and are interconnected using an IEEE- 488 bus, allowing utilization of standard measurement apparatus and peripherals. The system design allows user optimization of the measurement procedure, which can thus be tailored to meet specific experimental requirements. The fiexibility of this system is demonstrated by its application in spectroscopic ellipsometry.
\end{abstract}

\section{INTRODUCTION}

Although stand-alone automatic measuring systems are commercially available, to form an integrated system of two or more entails considerable expense and often problems arise connected with compatibility and duplication of components. The main advantage of our integrated system described here is that each of the modules, being of similar design, does not require significant additional expenditure and remains fully compatible. The resulting system avoids unnecessary duplication of components and provides maximum access to peripherals, e.g., plotter, printer, disk drive, etc.

Each module is capable of operating in both stand-alone and remote modes. In the latter case a main high-level language (HLL) program in the main computer controls the (external) modules via an IEEE-488 bus, permitting integration of several different measurement techniques using the same system.

\section{DESCRIPTION OF THE AUTOMATIC MEASURING SYSTEM}

The system can best be described in terms of three levels (Fig. 1). The first level is formed by the central computer, where the main (HCL) program resides, the IEEE-488 bus, and associated peripherals. The IEEE-488 bus allows maximum fiexibility in the choice of hardware, software, and existing measuring modules, e.g., digital multimeters, etc. The HLL program coordinates the measuring process, communicating with the external modules via specific handling procedures (handlers), and performs data processing and storage.

The second and third levels physically reside together in the external modules. The former corresponds to a sepurate microprocessor system, i.e., a position or voltage controller (PC or VC) or the data-acquisition system (DAS), having its own IEEE-488 interface and memory; the latter level contains a peripheral device specific to the module function.

\section{A. Position and voltage controllers}

Figures $2(a)$ and $2(b)$ show schematic diagrams of the main components associated with the second (microcontroller) and third (peripheral unit) levels of each module.
The microcontroller, based upon the $\mathrm{MC} 6802$ processor, receives its instructions from the main computer via the IEEE488 bus and interface GPIA MC68488; commands are subsequently passed on to the drive electronics via a set of optocouplers. In this fashion the microcontroller system is completely isolated from disturbances associated with electromagnetic induction in long connecting cables or spurious signals due to ground loops. Local control is realized via a keyboard input and display located on the internal microprocessor bus.

The peripheral unit (level 3) contains its own drive electronics, power supply, and drive unit (stepper motor or voltage output). This design permits the use of most commercially available three- and four-coil stepper motors. Replacement of the drive electronics by a digital-to-analog converter (DAC) makes the apparatus suitable for voltage regulation. In order to monitor the displacement in the PC

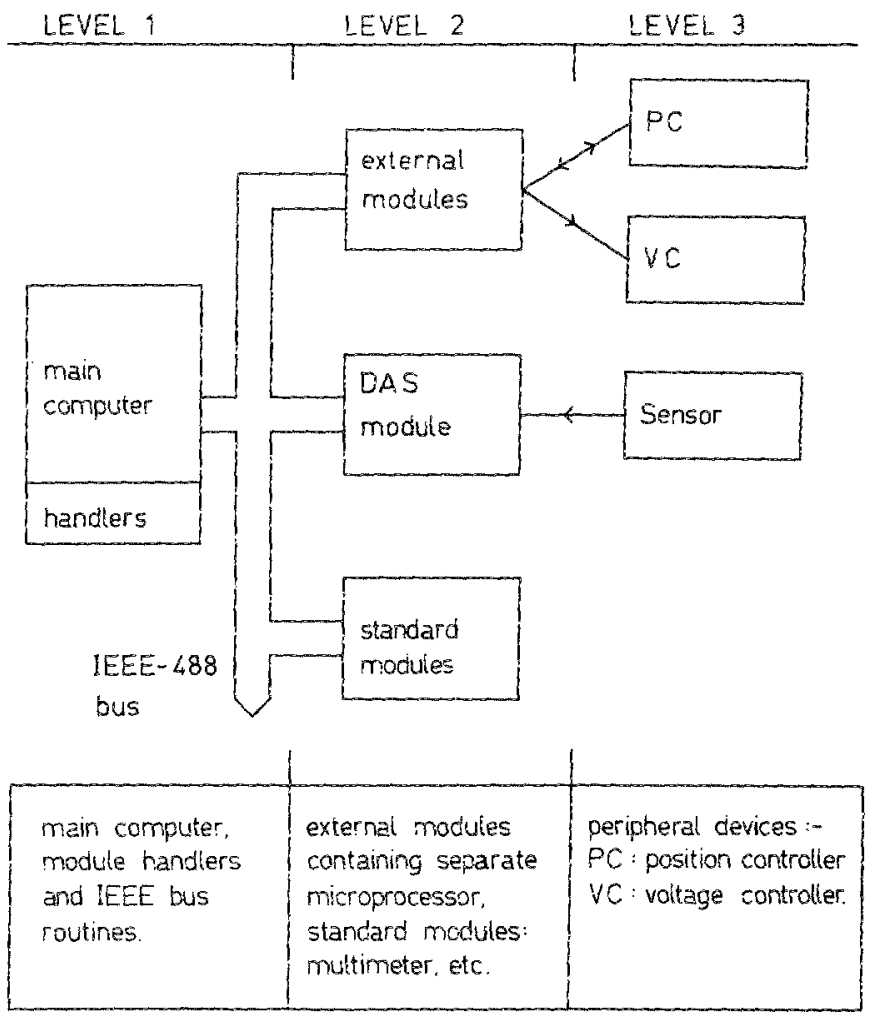

FIG. 1. Three levels of the modular measuring systern. 

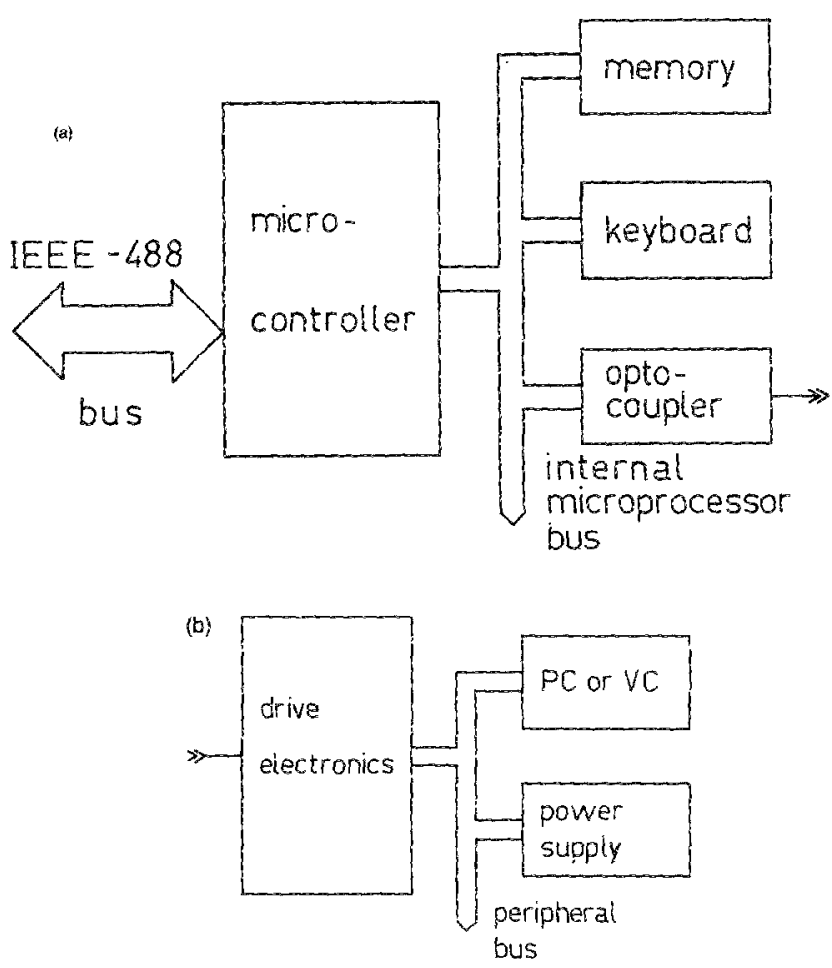

FIG. 2. (a) Second level (microcontroller); (b) third level (peripherai unit).

case, an encoder signal is fed back, via the optocoupler, to the microcontroller (indicated by the double arrow connecting the PC with the microcontroller in Fig. 1).

\section{B. Detalled description of the microcontroller (level 2)}

Figure 3 shows a schematic of the microcontroller, the heart of which is the microprocessor unit MC6802. Circuit diagrams for this controller are available ${ }^{1}$ but have been omitted for clarity; detailed component descriptions are availabie in the associated data manuals. ${ }^{2}$

We include here a brief description of the function of each chip. For the select and control logic, we have chosen a bipolar PROM MC6331 due to its combination of high packing density and flexibility of the memory map. The $1 \mathbb{K}$ RAM memory $91 \mathrm{~L} 24$, used for storage of, e.g., stack and program variables, was found to be large enough for most applications (excluding the DAS, which has RAM memory on a separate card).

The timer MC6840 contains three separate timers and is utilized for "time-out" interrupts and as the clock for the multiplexed display. Data exchange between the microcontroller and level 3 occurs via the PIA MC6821, a peripheral interface adaptor with optocoupler $1 / O$ card. The number of 1/O bits is user defined and depends upon application; e.g., the encoder signals from a stepper motor, or in the case of a DAC, data bits and data strobe signal.

The GPIA MC68488 is a general-purpose interface adaptor that controls communications protocol with level 1. The dip switch/buffer gives each module a unique IEEE-488 address upon power up. The GPIA completely implements

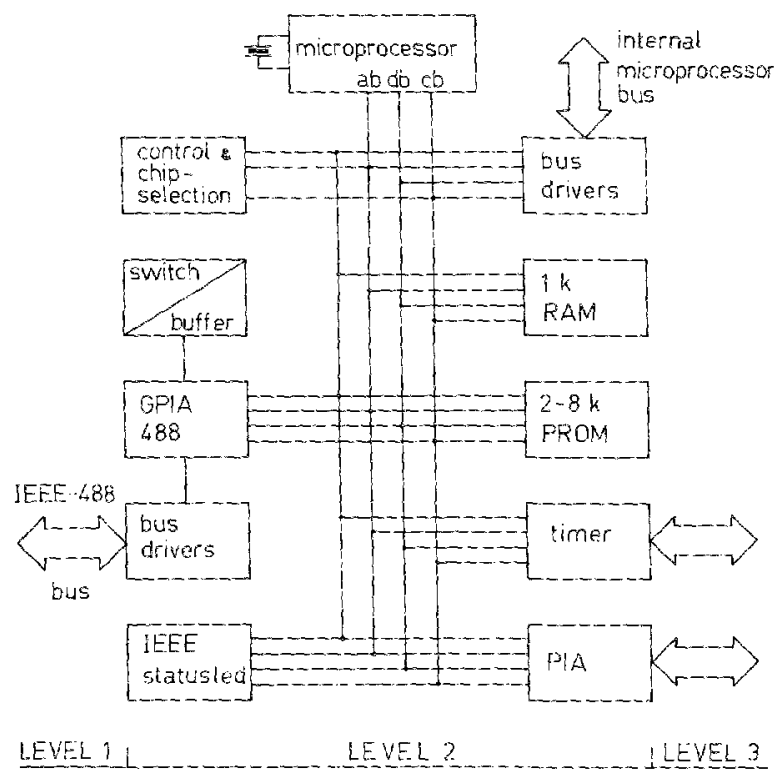

Frg. 3. Hiock schematic of the microcontroller. ad: address bus; db: data bus; cd: control bus.

all of the IEEE-488 standard interface functions (except that of controller) as well as providing special-purpose features; in conjunction with the MC3448 high-current bus driver, a complete IEEE-488 is realized. Five LEDs indicate the status of the interface, e.g., remote, listen, etc.

The bulk of the resident module program is stored in the 4K UV-PROM 2732. The program is the same in each module except for specific $1 / O$ routines, e.g., linear or rotary drive, voltage scan, etc. Each module microcontroller is identical, and all the components can be mounted on a single Euro card $\left(100 \times 160 \mathrm{~mm}^{2}\right)$. In addition, the buffered microprocessor bus section permits extension for more Euro cards and is used in our application for local control of the microcontroller via a keyboard and display. The display consists of $16 \times 7$-segment LEDs showing both the input parameters and current position, e.g., step size $(\mu \mathrm{m}, \mathrm{nm}, \mu \mathrm{V})$, trigger possibilities, scan speed, and scan mode (continuous, stepwise, or direct). The keyboard consists of two parts: numeric and a function input section for input of the initial values and steering modes described above.

We shall not treat the third level in such detail since it entirely depends upon the requirements, i.e., PC or VC. It should be mentioned, however, that much of the software is identical. We have constructed stepper motor drives for steering shutters, monochromators (and associated filter sets), rotation tables, and chopper wheels. In a similar fashion, 8-, 12-, and 16-bit DACs are used in several special applications, e.g., variabie reference voltage supplies, high-voltage sweep generators, and oven controllers. Detailed descriptions are available. ${ }^{3}$

We conciude the discussion of level 2 with a description of the DAS module. The DAS contains dual microprocessor systems MC6809 in a master/slave configuration (Fig. 4). Detailed descriptions of hardware ${ }^{1}$ and software $e^{4}$ are available, although the MC6809 card is very similar to the MC6802 that we have already discussed. The master/slave 


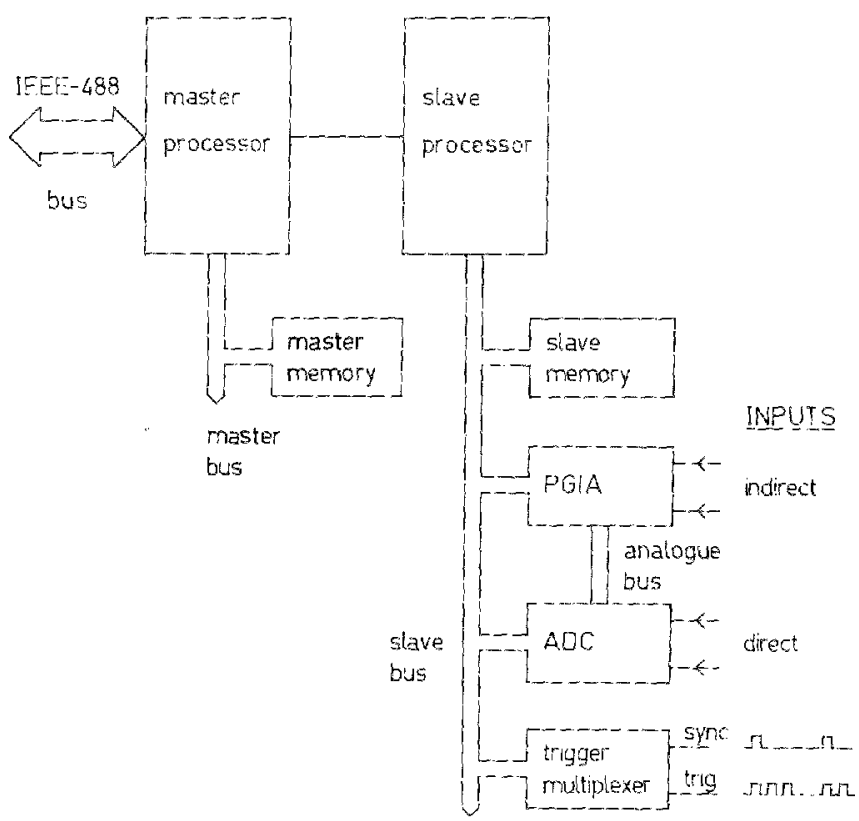

FIG. 4. Data-acquisition system (DAS).

configuration allows the master to perform other duties while the slave is measuring; as we will see (Sec. II), this arrangement is essential to optimize the overall performance of an integrated measuring system. The design requirements of the DAS included multichannel analog input ( 36 channels); large programmable gain range $(1,2,4, \ldots, 1024)$; fast data measurement, storage, and averaging; programmable filter on each input channel (rolloff $1,3,10, \ldots$, loK $\mathrm{Hz}$; ; and both internal and external triggering possibiiities.

The slave processor peripherals include memory [ 4 32) K RAM, 4K PROM], programmable gain instrumentation amplifier PGIA 3606BG (containing programmable filter), ADC 12-bit analogic MP6812, and trigger multiplexer.

The measurement parameters that the slave receives from the master processor contain information on slave data processing and slave peripheral settings (ADC, PGIA, etc. ). There are two DAS measuring modes, which, depending upon the type of triggering (external or internal), permit application of this system to the measurement of both periodic and nonperiodic signails. In the former case external synchronization (sync) and trigger inputs are required. We initially consider the external triggering mode, usually applied to periodic signals. Figure 5 illustrates the slave measuring cycle: the incoming signal is sampled $P$ times per cycle, and each discrete signal height is stored in a memory array location. During the next period, the new signal height is added to the appropriate array location. Upon completion of the measurement, the contents of each memory location are divided by the number of loops.

The above array integration technique has two important advantages: First, noise signals having a period different from that of the measurement period are quickly averaged out; indeed for random noise the accuracy is smaller than the bit resolution $(2.5 \mu \mathrm{V}$ at gain $=1024)$. Second, this method

$$
\begin{aligned}
& \text { Slave } \\
& \text { memory } \\
& \text { map }
\end{aligned}
$$
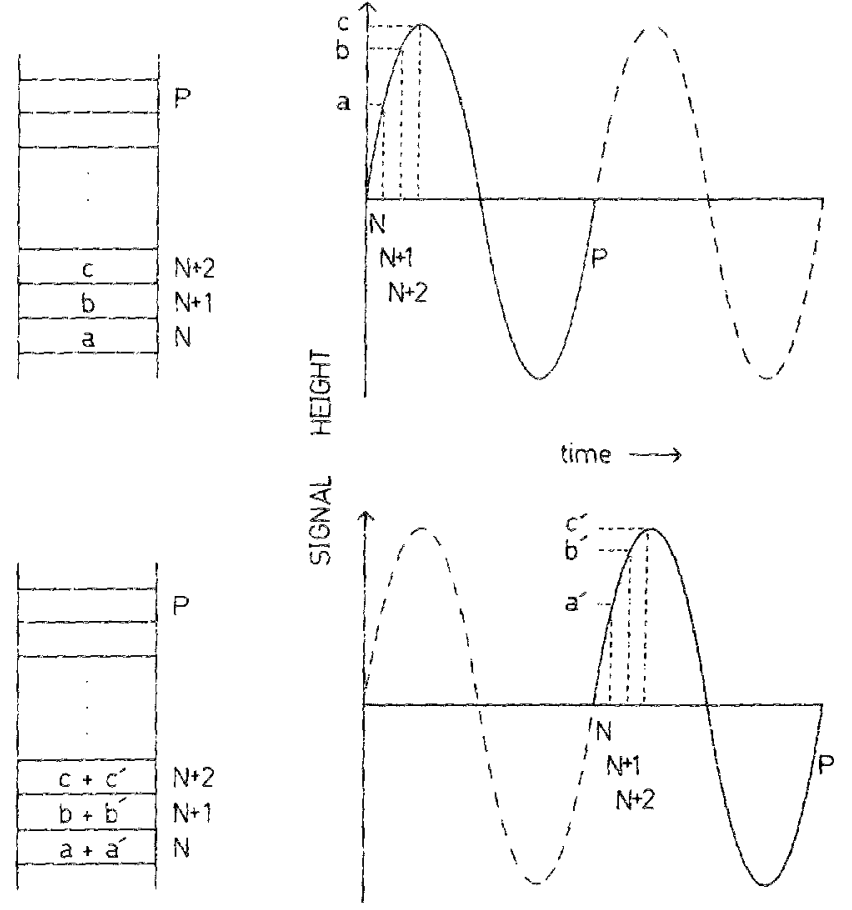

Fic. 5. Slave signal measurement and averaging (external triggering).

of integration (boxcar infegration) can be quickly performed ( $80 \mu$ s per measured point), allowing the signal to be analyzed as a continuous data stream.

In the second measuring mode, utilizing internal triggering, the slave measures one data point. Signal averaging is accomplished in a similar fashion to that described above, with an array length of 1.

The DAS also contains a display showing the channel number, gain, and filter settings plus the presence of trigger signals. This addition has proved to be an invaluable aid in fault diagnosis.

\section{INTEGRATED AUTOMATIC MEASURING SYSTEM APPLIED TO SPECTROSCOPIC ELLIPSOMETRY}

The principles of the ellipsometer reported here are based on the Fourier analysis method described by various authors. ${ }^{5,6}$ Our setup is illustrated in Fig. 6: light from a broadband halogen source passes first through a grating monochromator and filter arrangement and then through a polarizer before striking the sample. The reflected beam is modulated by a rotating analyzer (rotan) before arriving at the detector. by

The output intensity $I$ of the detector can be described

$$
I=k_{0}+k_{1} \cos 2 A+k_{2} \sin 2 A,
$$

where $k_{0}, k_{1}$, and $k_{2}$ are the Fourier coefficients of the signal and $A$ is the azimuth angle of the analyzer. The ellipsometric parameters $\Delta$ and $\psi$ can then be calculated from the $k$ 's. 


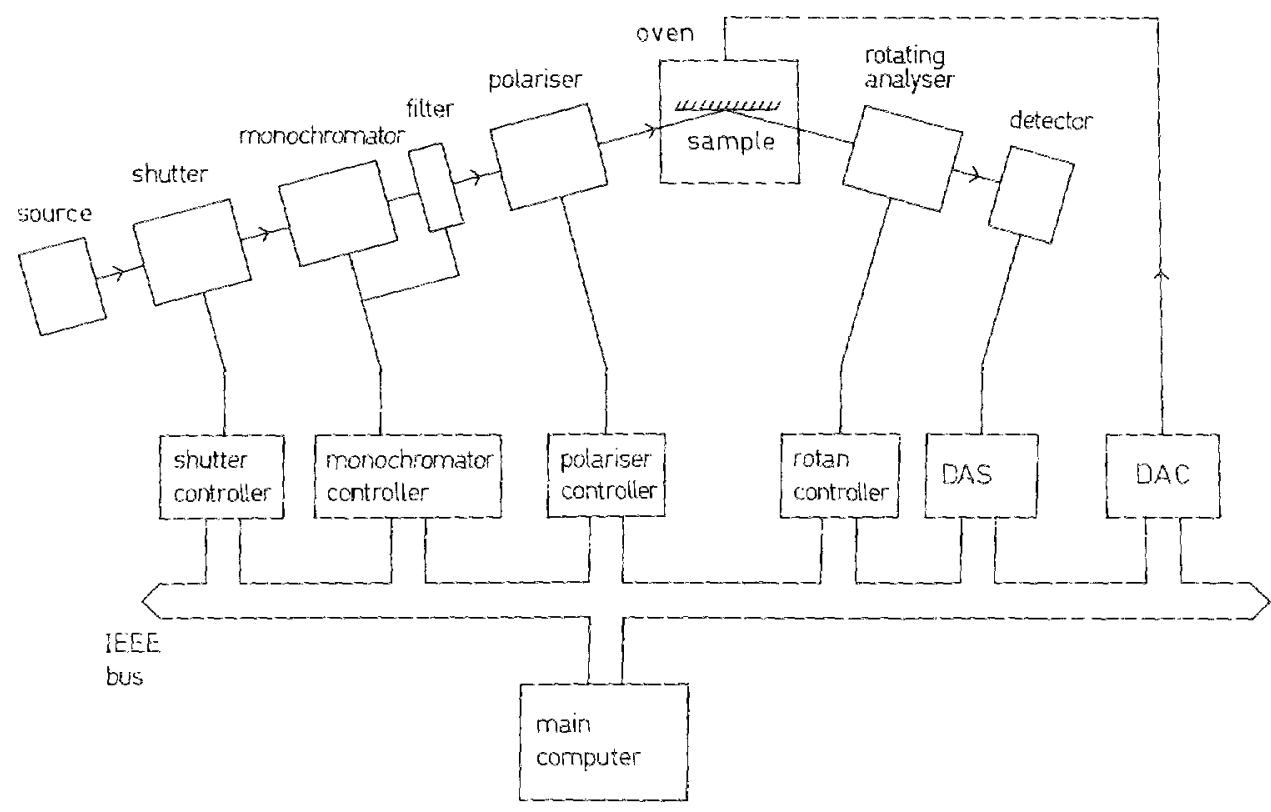

FI. 6. Spectroscopic ellipsometer system.

Thus any changes in $\Delta$ and $\psi$ are refected in corresponding changes in the $k$ 's.

Six external modules are used in the configuration described above: the shutter, monochromator, polarizer and rotan controllers, a DAC for temperature regulation, and the DAS. The DAS measures in the external triggering mode: the sync and trigger pulses are generated by a disk/encoder system attached to the rotating analyzer, producing 1 sync and 180 trigger puises per revolution.

The measurement cycle proceeds as follows: the initial module paraneters, e.g., sample temperature, monochromator wavelength, polarizer angle, loops, filter, gain, ete., are installed via the handlers at level 1 . Once the required set of conditions is attained, a dark-light measurement (see below) is performed using the shutter, monochromator, and DAS modules. After the measurement data have been transferred to the main computer, a new set of input parameters is then passed to the various microcontroliers. During seiting of these parameters, the main computer can proceed with data processing; e.g., the data are corrected for offset, the Fourier components and the $(\Delta, \psi)$ computed and stored.

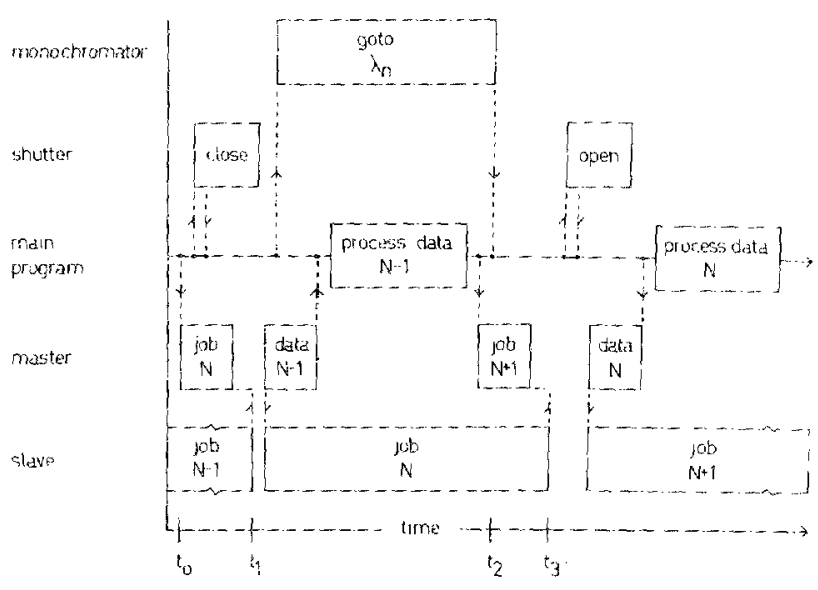

Frg. 7. Dark-light measurement.
We consider the dark-light loop of the above measurement cycle in order to illustrate the interaction between the master/slave configuration, the microcontrollers, and the main computer. The aim is to optimize the time the slave is working. The measurement loop (Fig. 7) is as follows: at time $t_{0}$ the main program passes the $N$ th job parameters to the master, then closes the shutter. At $t$, the slave completes the ( $N-1$ ) th job, sends the measured array to the master, receives the $N$ th job parameters, and begins measurement (on the "dark" signal).

The main program now instructs the monochromator to go to wavelength $\lambda_{N}$, then processes the $(N-1)$ th job data. At time $t_{2}$ the monochromator module indicates that the monochromator is in position. Shortly afterward at $t_{3}$, the $N$ th job is finished, the main program opens the shutter, and the master stores the slave array, submits the $(N+1)$ th job to the slave, and finally passes the $N$ th job data to the main computer for processing.

The above dark-light measurement loop is used in most optical measurement systems to eliminate stray light and detector offset signals. This parallel job-handing technique is used extensively throughout the ellipsometer program, allowing the user to optimize the performance of the measuring system.

\section{ACKNOWLEDGMENTS}

One of the authors (Baikie) was the holder of a scholarship from the Science and Engineering Research Council, Swindon, England. The authors would like to thank W. Vonk and R. J. S. Sieverdink for their assistance in the construction of the modules.

'K. O. van der Werf, Internal Technical Report No. MC86-1, University of Twente, 1986.

${ }^{2}$ Motorola Microprocessor Data Manual, 1982.

${ }^{3}$ E. G. Keim, Surf. Sci. 180, 565 (1987).

${ }^{4} \mathrm{H}$. Bloem and R. J. S. Sieverdink, Internal Technical Report No. MC86-2, University of Twente, 1986.

${ }^{5}$ D. E. Aspnes and A. A. Studna, Appl. Opt. 14, 220 (1975).

'R. M. A. Azzam and N. M. Bashara, Ellipsometry and Polarized Light (North-Holland, Amsterdam, 1977). 